\title{
Comment on "Theoretical Analysis and Circuit Verification for Fractional-Order Chaotic Behavior in a New Hyperchaotic System"
}

\author{
Jay Prakash Singh and B. K. Roy \\ National Institute of Technology, Silchar, Silchar 788010, India \\ Correspondence should be addressed to Jay Prakash Singh; jayprakash1261@gmail.com
}

Received 10 February 2016; Accepted 30 June 2016

Academic Editor: Dan Simon

Copyright (C) 2016 J. P. Singh and B. K. Roy. This is an open access article distributed under the Creative Commons Attribution License, which permits unrestricted use, distribution, and reproduction in any medium, provided the original work is properly cited.

Some comments on the paper "Theoretical Analysis and Circuit Verification for Fractional-Order Chaotic Behavior in a New Hyperchaotic System" (L. Liu and C. Liu, 2014) are pointed out in this letter. It is shown in this letter that the claimed hyperchaotic system exhibits a periodic behaviour for the chosen parameters and initial condition. However, the claimed hyperchaotic system exhibits chaotic behaviours for some other parameters.

The dynamics of the hyperchaotic system considered in [1] is as follows:

$$
\begin{aligned}
& \dot{x}_{1}=\beta_{1}\left(x_{2}+0.2\left(x_{1}-2 x_{1}^{3}\right)\right), \\
& \dot{x}_{2}=\beta_{2} x_{1}-x_{2}+x_{3}+x_{4}, \\
& \dot{x}_{3}=-\beta_{3} x_{2}+x_{4}, \\
& \dot{x}_{4}=-\beta_{4} x_{1} .
\end{aligned}
$$

It is mentioned in [1] that system (1) is hyperchaotic with $\beta_{1}=10, \beta_{2}=1, \beta_{3}=12.5$, and $\beta_{4}=5$. The initial condition $x(0)=(0.2,0.05,0.08,0)^{T}$ was used for simulation. The strange attractors of the system were shown in Figure 2 of [1]. The Lyapunov exponents were calculated as $(0.2458,0.0175,-0.0398,-3.2881)$.

We simulated system (1) for the same parameters and initial condition. The results are shown in Figure 1. Figure 1 shows periodic behaviour of (1) in contrast with hyperchaotic behaviour as claimed in [1]. It was mentioned in [1] that the Wolf et al. [2] algorithm was used for calculation of the Lyapunov exponents. We have also used the same algorithm to calculate the Lyapunov exponents with different sampling time and observation time, and the results are listed in Table 1. The plots of Lyapunov exponents of system (1) for 10000 observation times with 0.02 sampling time for $\beta_{1}=10$, $\beta_{2}=1, \beta_{3}=12.5$, and $\beta_{4}=5$ are shown in Figure 2. Thus, the Lyapunov exponents (Table 1 and Figure 2) reveal that system (1) has periodic behaviour for the chosen parameters and initial condition. It may be noted that the Lyapunov exponents of system (1) with the chosen parameters and initial condition are of $(\approx 0,-,-,-)$ nature which is in contrast with $(+,+,-,-)$ as claimed in [1].

However, the claimed hyperchaotic system in [1] behaves in a chaotic manner for some other value of the parameters. These parameters were used for calculating the eigenvalues in [1]. The attractors of system (1) for $\beta_{1}=8.25, \beta_{2}=0.8$, $\beta_{3}=12.25$, and $\beta_{4}=0.2$ are shown in Figure 3. The Lyapunov exponents are calculated for different sampling steps and observation times and are listed in Table 2. System (1) has an unusual chaotic behaviour with $(+, \approx 0, \approx 0,-)$ nature of Lyapunov exponents. This nature of LEs for chaotic systems is rare in the literature, although such nature of Lyapunov exponents leads to chaotic behaviour as claimed in [3]. Figure 3 validates the chaotic behaviour of system (1).

Finally, the claim of the hyperchaotic system for the chosen parameters and the initial condition by authors in [1] could not be established through our numerical simulations. 


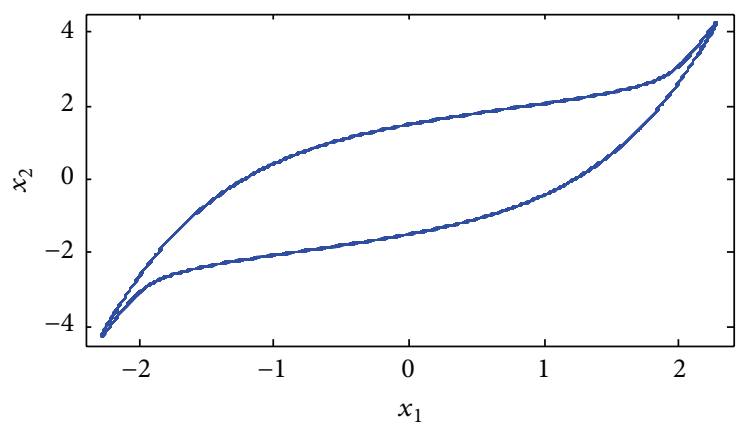

(a)

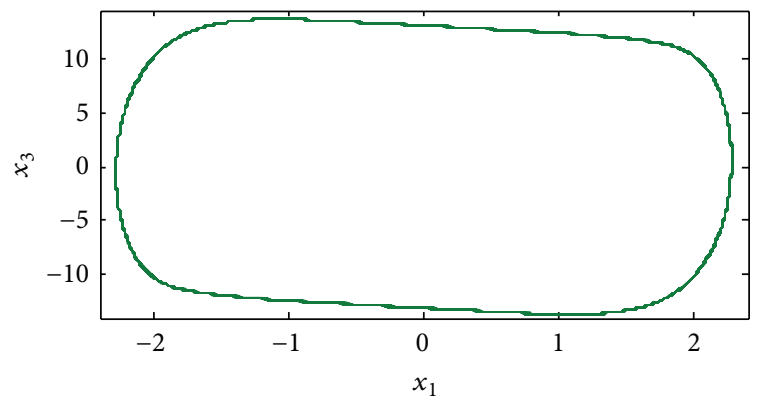

(c)

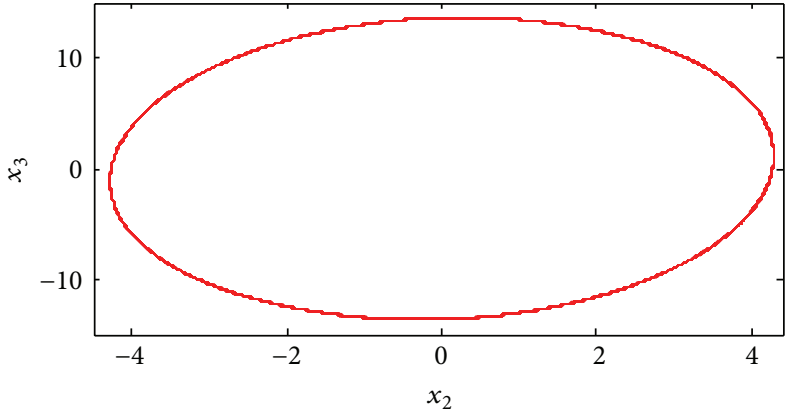

(b)

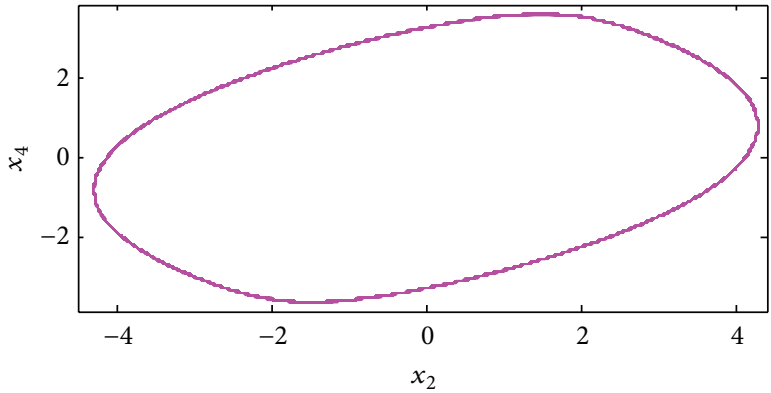

(d)

FIGURE 1: Phase portraits of system (1) with $\beta_{1}=10, \beta_{2}=1, \beta_{3}=12.5$, and $\beta_{4}=5$ : (a) on $x_{1}-x_{2}$ plane, (b) on $x_{2}-x_{3}$ plane, (c) on $x_{1}-x_{3}$ plane, and $(\mathrm{d})$ on $x_{2}-x_{4}$ plane for $x(0)=(0.2,0.05,0.08,0)^{T}$.

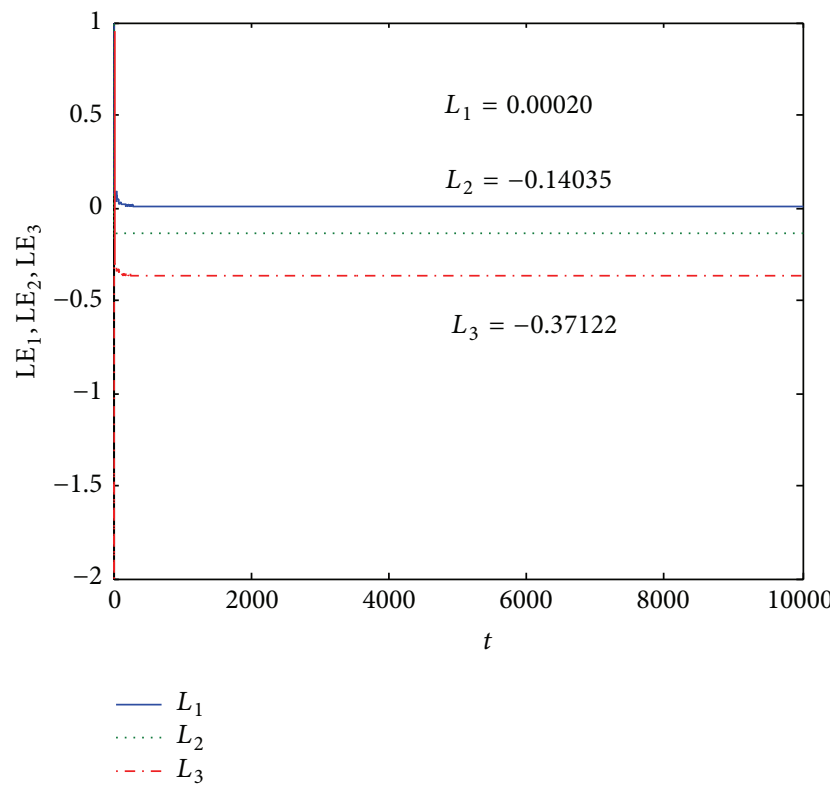

(a)

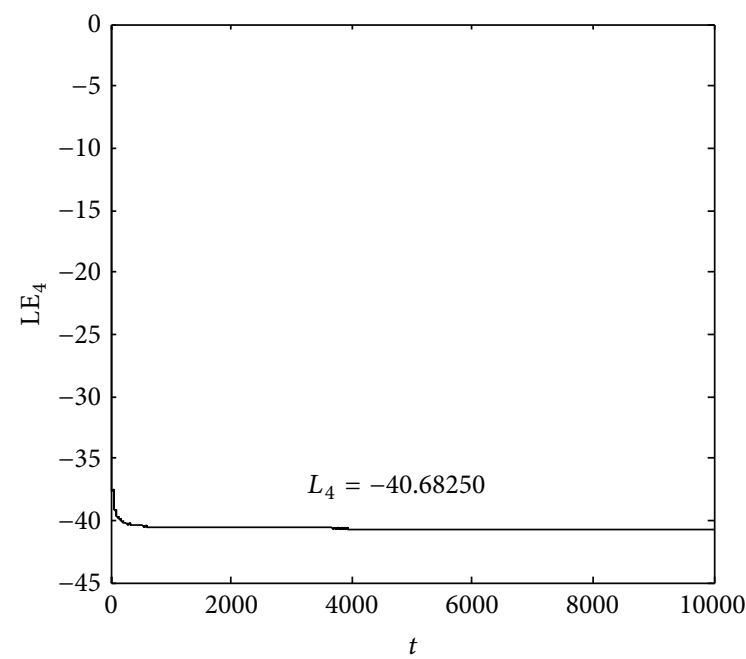

FIGURE 2: Lyapunov exponents of the system for $\beta_{1}=10, \beta_{2}=1, \beta_{3}=12.5$, and $\beta_{4}=5$ with $\Delta t=0.02$ and $x(0)=(0.2,0.05,0.08,0)^{T}$.

TABLE 1: Lyapunov exponents of system (1) with different sampling and observation times for $\beta_{1}=10, \beta_{2}=1, \beta_{3}=12.5$, and $\beta_{4}=5$.

\begin{tabular}{|c|c|c|c|c|}
\hline Sl. number & Initial condition & Time & Step size & Lyapunov exponents \\
\hline 1 & $0.2,0.05,0.08,0$ & 500 & 0.02 & $0.0050,-0.1406,-0.3663,-40.4492$ \\
\hline 2 & $0.2,0.05,0.08,0$ & 300 & 0.05 & $0.0033,-0.1401,-0.3692,-40.5024$ \\
\hline 3 & $0.2,0.05,0.08,0$ & 1000 & 0.02 & $0.0028,-0.1405,-0.3671,-40.5025$ \\
\hline 4 & $0.2,0.05,0.08,0$ & 10000 & 0.02 & $0.0002,-0.1403,-0.3710,-40.5025$ \\
\hline
\end{tabular}


TABLE 2: Lyapunov exponents of system (1) with $\beta_{1}=8.25, \beta_{2}=0.8, \beta_{3}=12.25$, and $\beta_{4}=0.2$ for different sampling and observation times.

\begin{tabular}{|c|c|c|c|c|}
\hline Sl. number & Initial condition & Time & Step size & Lyapunov exponents \\
\hline 1. & $0.2,0.05,0.08,0$ & 2000 & 0.002 & $0.1350,0.0022,-0.0077,-2.9123$ \\
\hline 2. & $0.2,0.05,0.08,0$ & 1000 & 0.02 & $0.1184,0.0079,-0.0079,-2.940$ \\
\hline 3. & $0.2,0.05,0.08,0$ & 5000 & 0.02 & $0.1256,0.0018,-0.0084,-2.9243$ \\
\hline 4. & $0.2,0.05,0.08,0$ & 10000 & 0.02 & $0.1251,0.0007,-0.0086,-2.9121$ \\
\hline
\end{tabular}

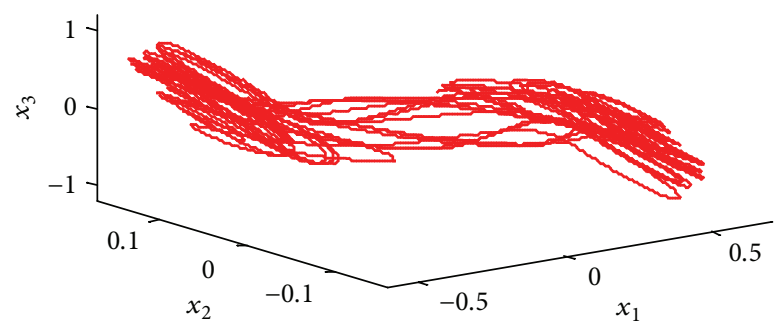

(a)

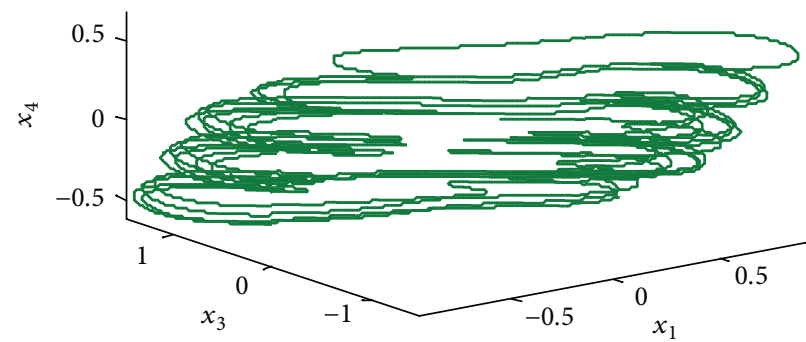

(c)

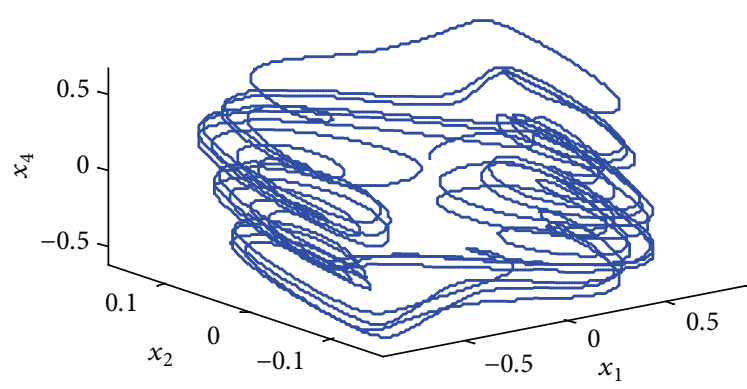

(b)

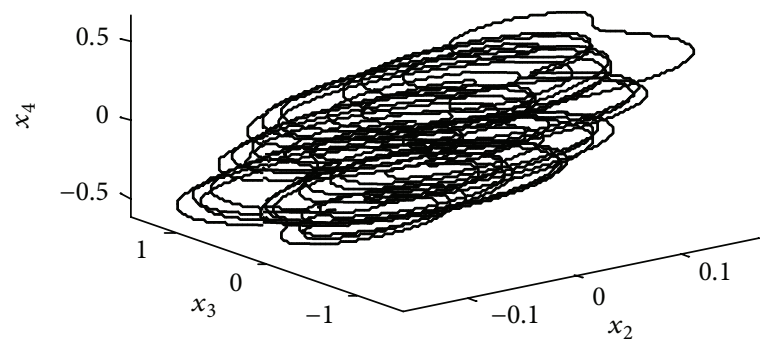

(d)

FIGURE 3: Chaotic attractors of system (1) with $\beta_{1}=8.25, \beta_{2}=0.8, \beta_{3}=12.25$, and $\beta_{4}=0.2$ : (a) in $x_{1}-x_{2}-x_{3}$ space, (b) in $x_{1}-x_{2}-x_{4}$ space, (c) in $x_{1}-x_{3}-x_{4}$ space, and (d) in $x_{2}-x_{3}-x_{4}$ space.

\section{Competing Interests}

The authors declare that they have no competing interests.

\section{References}

[1] L. Liu and C. Liu, "Theoretical analysis and circuit verification for fractional-order chaotic behavior in a new hyperchaotic system," Mathematical Problems in Engineering, vol. 2014, Article ID 682408, 14 pages, 2014.

[2] A. Wolf, J. B. Swift, H. L. Swinney, and J. A. Vastano, "Determining Lyapunov exponents from a time series," Physica D: Nonlinear Phenomena, vol. 16, no. 3, pp. 285-317, 1985.

[3] M. H. Mohammadinejad and H. M. Moslehi, "Continious attractors in hopfiled neural networks," International Journal of Differential Equations and Applications, vol. 13, no. 2, pp. 61-70, 2014. 


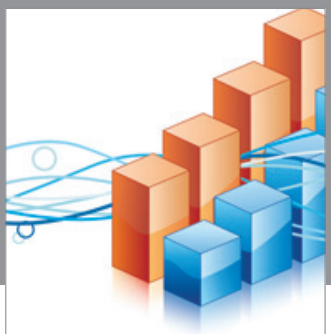

Advances in

Operations Research

vatem alat4

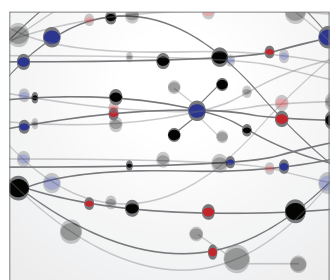

\section{The Scientific} World Journal
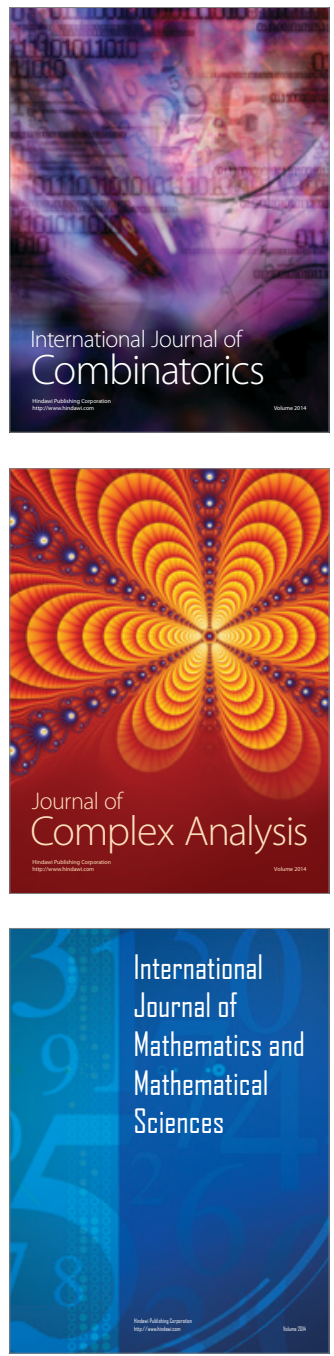
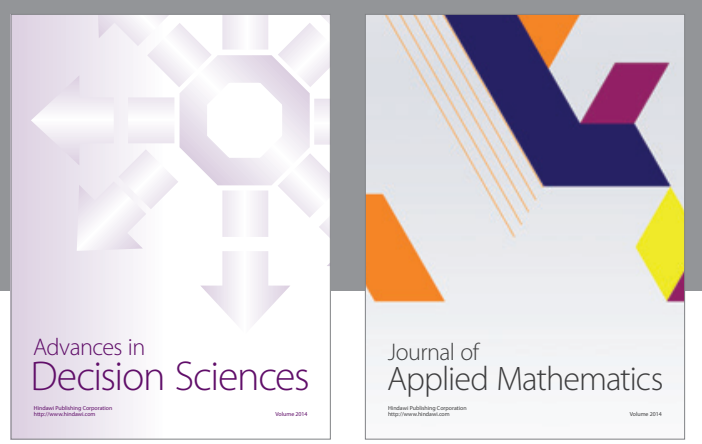

Algebra

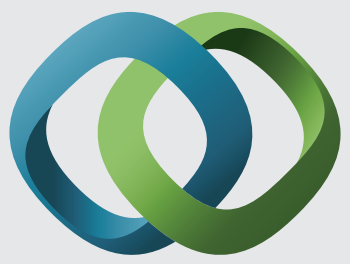

\section{Hindawi}

Submit your manuscripts at

http://www.hindawi.com
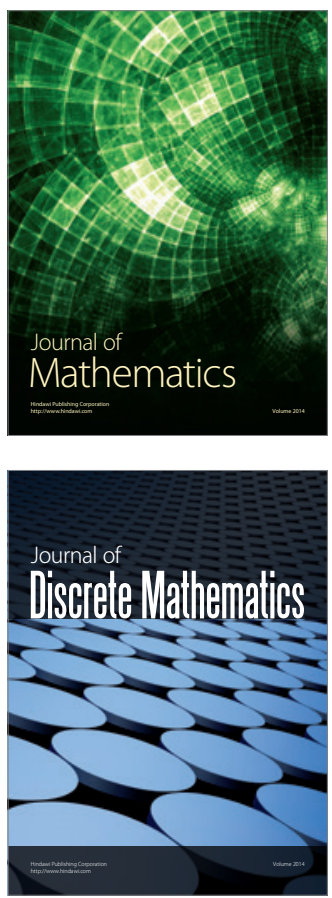

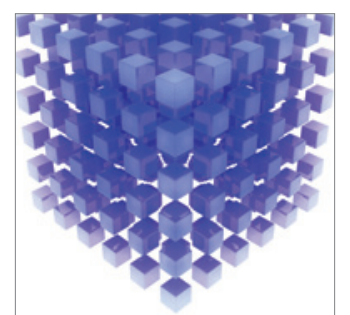

Mathematical Problems in Engineering
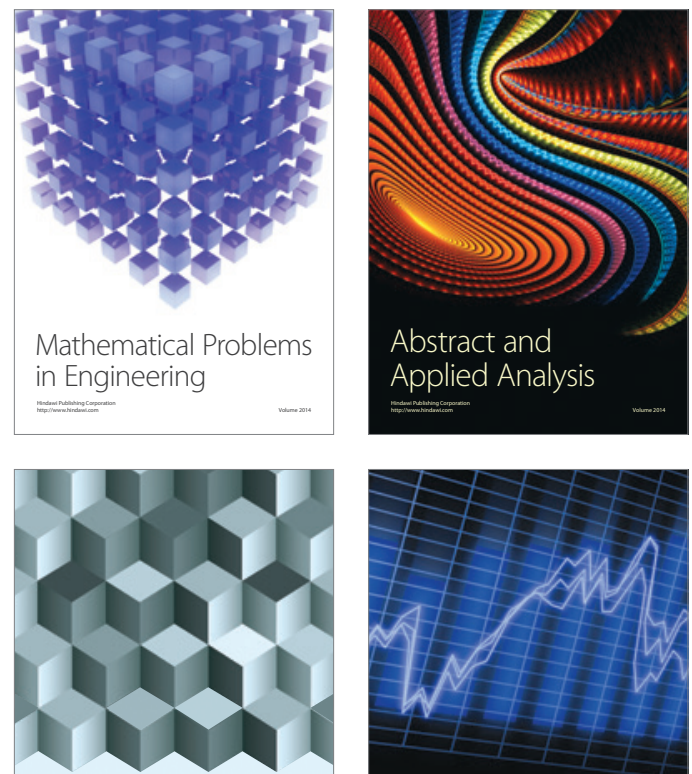

Journal of

Function Spaces

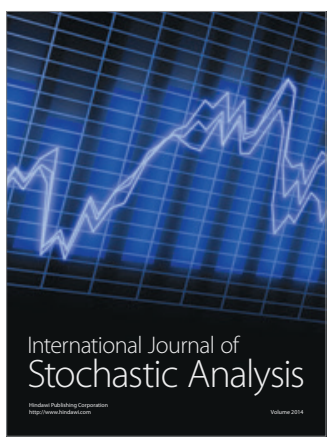

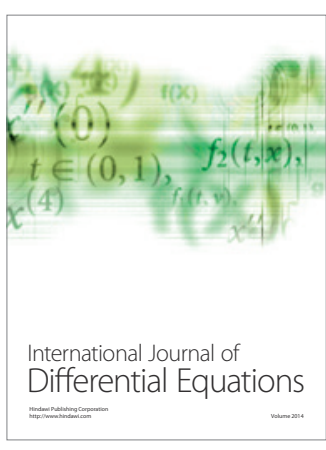
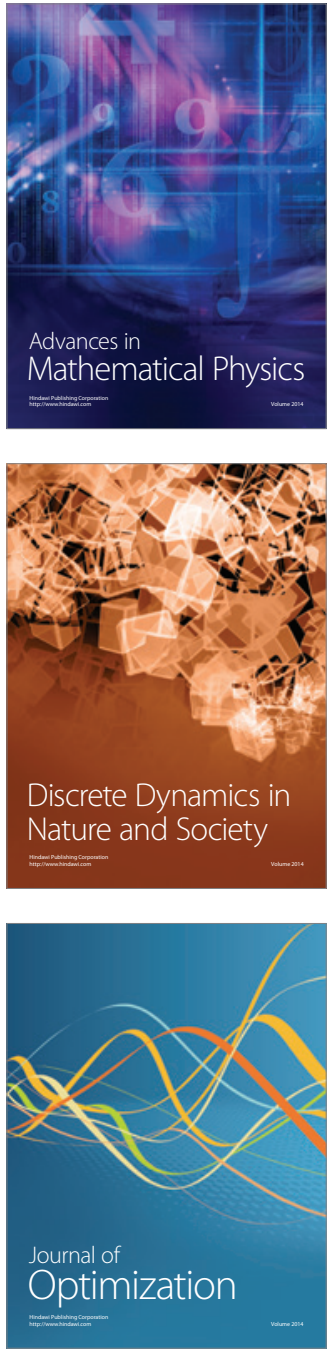